\title{
Bandpass transmission filters based on phase shifted fiber Bragg gratings in microstructured polymer optical fibers
}

Ortega, Beatriz; Min, Rui; Sáez-Rodri-guez, David; Mi, Yang; Nielsen, Kristian; Bang, Ole

Published in:

Proceedings of SPIE

Link to article, DOI:

$10.1117 / 12.2268281$

Publication date:

2017

Document Version

Publisher's PDF, also known as Version of record

Link back to DTU Orbit

Citation (APA):

Ortega, B., Min, R., Sáez-Rodri-guez, D., Mi, Y., Nielsen, K., \& Bang, O. (2017). Bandpass transmission filters based on phase shifted fiber Bragg gratings in microstructured polymer optical fibers. In Proceedings of SPIE (Vol. 10232). [1023209] SPIE - International Society for Optical Engineering. Proceedings of SPIE - The International Society for Optical Engineering https://doi.org/10.1117/12.2268281

\section{General rights}

Copyright and moral rights for the publications made accessible in the public portal are retained by the authors and/or other copyright owners and it is a condition of accessing publications that users recognise and abide by the legal requirements associated with these rights.

- Users may download and print one copy of any publication from the public portal for the purpose of private study or research.

- You may not further distribute the material or use it for any profit-making activity or commercial gain

- You may freely distribute the URL identifying the publication in the public portal 


\author{
Bandpass transmission filters based on phase shifted fiber Bragg \\ gratings in microstructured polymer optical fibers \\ Beatriz Ortega*a $^{*}$ Rui Min ${ }^{\mathrm{a}}$, David Sáez-Rodriguez ${ }^{\mathrm{a}}$, Yang $\mathrm{Mi}^{\mathrm{a}}$, Kristian Nielsen ${ }^{\mathrm{b}, \mathrm{c}}$, Ole Bang ${ }^{\mathrm{b}, \mathrm{c}}$ \\ ${ }^{a}$ ITEAM Research Institute, Universitat Politècnica de Valencia, Valencia, Spain 46022; \\ ${ }^{b}$ DTU Fotonik, Department of Photonics Engineering, Technical University of Denmark, 2800 Kgs, \\ Lyngby, Denmark \\ ${ }^{\mathrm{c}}$ SHUTE Sensing Solutions, 2800 Kongens Lyngby, Denmark
}

\begin{abstract}
In this contribution we report on the fabrication of novel bandpass transmission filters based on PS-FBGs in microstructured polymer fibers at telecom wavelengths. The phase mask technique is employed to fabricate several superimposed gratings with slight different periods in order to form Moiré structures with a single or various $\pi$ phase shifts along the device. Simulations and experimental results are included in order to demonstrate very narrowband transmission filters. Experimental characterization under strain and temperature variations is provided in a non-annealed fiber and time stability of the fabricated devices has been also measured under different pre-strain conditions.
\end{abstract}

Keywords: Polymer optical fibers, fiber Bragg grating, optical fiber devices, optical filters

\title{
1. INTRODUCTION
}

Phase-Shifted fiber Bragg gratings (PS-FBG) in silica fibers have already been demonstrated during the last decade as attractive very narrow filters for a variety of applications such as real-time monitoring, switching, and demodulating [14]. Special attention must be paid to the sensing field, where PS-FBGs find applications to detect ultrasound [5], pressure [6], and temperature [7], among others. Moreover, very recently published papers report that PS-FBG also find applications in the microwave photonics field, such as a tunable bandstop-to-bandpass microwave photonic filter [8] or a high resolution strain interrogation system based on two phase-shifted FBGs in a dual frequency optoelectronic oscillator [9]. Moreover, as reported in the previous literature, multiple phase shifts can be used to tailor the transmission spectrum for wavelength demultiplexing in DWDM applications [10-12].

Polymer optical fibers (POFs) have several advantages over silica fibers, such as a larger elongation before breakage, a higher thermo-optic coefficient, and a lower Young's modulus. These advantages will provide a broad tuning range either straining or heating the fiber, and easy handling due to its low stiffness and therefore, lower installations cost. However, the product of bandwidth and channel length is somewhat reduced due to higher loss [13] and they are suitable for in-building and short range applications.

Among their current applications, POF sensors apply to a large variety of areas, such as deformation and pressure sensors [14], humidity sensors [15], biosensors [16], etc. In the field of telecommunications, POF is used for short distance connections such as in-vehicle networks (cars, trains or air planes), local area networks (offices, homes or buildings) and interconnection (onboard or intraboard) [17]. Recently, the advancement of new Cytop fibers with reduced losses at telecom wavelengths has led to new promising applications [18].

Currently, one of the challenges of POF systems is to increase the capacity while QoS is assured in current and future services. Present communication systems over POF use a single channel for data transmission, but in recent years, there is a growing interest to develop wavelength division multiplexed (WDM) systems in order to increase the maximum data rate over a single fiber keeping the mentioned advantages [19-21]. In such networks, either bulk devices and/or couplers are employed to mux/demux different channels [20]. Fiber Bragg Gratings in POF are required to obtain compact and low cost mux/demux components in WDM-POF networks along medium and short telecommunications distances. Moreover, as POF networks demands higher capacity due to the rapid growth of high bandwidth applications, i.e. data storage, high definition video or fast internet access amongst others, the need of devices which can mux/demux several channels in order to route them to different sub-networks is becoming increasing.

\footnotetext{
Micro-structured and Specialty Optical Fibres V, edited by Kyriacos Kalli, Jiri Kanka, Alexis Mendez,

Pavel Peterka, Proc. of SPIE Vol. 10232, 1023209 - ㅇ 2017 SPIE

CCC code: $0277-786 \mathrm{X} / 17 / \$ 18 \cdot$ doi: $10.1117 / 12.2268281$
} 
FBGs in silica fibers is a very mature filtering technology both for telecommunications and sensors since a large variety of high performance FBGs can be fabricated by creating a UV light induced periodic variation in the refractive index of the fiber core. However, since the first polymer FBG reported in 1999 [22], several promising devices, mainly for the measurement of strain and temperature using step-index fiber and micro-structured fiber have been demonstrated [2325]. Despite of this, for the inscription of the grating, the photosensitivity of the material is important; so far great advances have been done both in doped [26-28] and undoped fibers [29], but grating time stability is still an issue if the FBG is not properly annealed [24,30]. Previous literature reports that power density have an effect on time stability of the grating [31] and also a chirped Bragg grating inscribed using an $\mathrm{KrF}$ excimer laser at 248nm kept unchanged for 13 weeks [32].

Regarding phase-shifted FBGs in POFs, the literature only reports a device, which was for use at $\mathrm{THz}$ frequencies, fabricated by using a point-by-point technique [33]. In this paper, to the best of our knowledge, we report on the fabrication of FBG with one or more phase shifts in polymer fibers by using the phase mask technique. Two gratings with slight different periods have been superimposed in the fiber in order to form a Moire structure with a $\pi$ phase shift in the center of the device [34-35]. Simulations have been done to predict the response of the fabricated structures and very narrowband transmission filters are fabricated. Experimental characterization under strain and temperature variations is provided and also time stability of the devices has been included.

\section{SIMULATION OF PS-FBG STRUCTURES}

A phase shifted FBG is obtained by the insertion of one or more phase shifts along the grating. Non-uniform gratings are modeled by the piecewise-uniform approach which consists in identifying a $2 \times 2$ matrix for each uniform section of the grating and multiplying all of them to obtain the response of the whole grating. Provided the grating is divided in $\mathrm{M}$ uniform sections, its output and input fields are related by the cascaded product of the transmission matrices corresponding to the different sections, as follows [36]:

$$
\left[\begin{array}{c}
A_{\text {out }} \\
B_{\text {out }}
\end{array}\right]=F_{M} \cdot F_{M-1} \cdots \cdot F_{i} \cdots \cdot F_{1}\left[\begin{array}{c}
A_{\text {in }} \\
B_{\text {in }}
\end{array}\right]
$$

Where $\mathrm{A}$ and $\mathrm{B}$ represent the field amplitudes travelling in the $+\mathrm{z}$ and $-\mathrm{z}$ directions, respectively. The required accuracy for correct calculation determines the number of sections. The matrix element $F_{i}$ is obtained by the standard coupled mode theory:

$$
\mathrm{F}_{i}=\left[\begin{array}{cc}
\cosh \left(\gamma_{\mathrm{B}} \Delta \mathrm{z}\right)-\mathrm{i} \frac{\hat{\sigma}}{\gamma_{\mathrm{B}}} \sinh \left(\gamma_{\mathrm{B}} \Delta \mathrm{z}\right) & -\mathrm{i} \frac{\kappa}{\gamma_{\mathrm{B}}} \sinh \left(\gamma_{\mathrm{B}} \Delta \mathrm{z}\right) \\
\mathrm{i} \frac{\kappa}{\gamma_{\mathrm{B}}} \sinh \left(\gamma_{\mathrm{B}} \Delta \mathrm{z}\right) & \cosh \left(\gamma_{\mathrm{B}} \Delta \mathrm{z}\right)-\mathrm{i} \frac{\hat{\sigma}}{\gamma_{\mathrm{B}}} \sinh \left(\gamma_{\mathrm{B}} \Delta \mathrm{z}\right)
\end{array}\right]
$$

where $\Delta \mathrm{z}$ is the length of the ith section, the coupling coefficients $\widehat{\sigma}$ and $\kappa$ are the local values in the ith section, and also:

$$
\gamma_{\mathrm{B}}=\sqrt{\kappa^{2}-\widehat{\sigma}^{2}}
$$

\subsection{Matrix method for PS-FBG}

The simulation of the response of a single phase shifted grating can be easily done by inserting a phase shift matrix, $\mathrm{F}_{\mathrm{ps}}$, between the matrices $F_{i}$ anf $F_{i+1}$ for a phase shift just after the ith section. In this case, the FBG is composed of three sections which correspond to two uniform sections and between them, one section corresponding to the phase shift. The phase shift transfer matrix is expressed as:

$$
\mathrm{F}_{\mathrm{ps}}=\left[\begin{array}{cc}
\mathrm{e}^{-i \phi / 2} & 0 \\
0 & \mathrm{e}^{i \phi / 2}
\end{array}\right]
$$

where $\phi$ is the shift in the phase of the grating for discrete phase shifts.

Figure 1 shows the reflectivity and phase response of a $2.5 \mathrm{~mm}$ long grating with $\pi$ (Figures 1.a), c) and e)) and $\pi / 2$ (Figures 1.b), d) and f)) phase shift at three different positions along the grating: central, $-\mathrm{L} / 6$ and $+\mathrm{L} / 6$ respect to the centre of the grating, respectively. The refractive index of the fiber is 1.46 and the grating period $\Lambda_{1}$ is $533.1 \mathrm{~nm}$. The reflection notch of Figure 1a) shows a $3 \mathrm{~dB}$ bandwidth of $0.047 \mathrm{~nm}$. 
Figure 2a) shows the reflectivity and phase response of the grating when two pi phase shifts are equispaced along the grating and three identical uniform sections are defined, and similarly, Figure 2b) shows the response of a grating including three pi phase shifts which lead to four identical sections. In this case, the simulated $3 \mathrm{~dB}$ bandwidth of the notch encountered in reflection is about $0.66 \mathrm{~nm}$. Phase responses in all cases allow to confirm the structure we are simulating. In the following section we will show the theoretical equivalence of a Moiré structure, which will allow us to do proper design of gratings to be inscribed by using a simple fabrication setup.
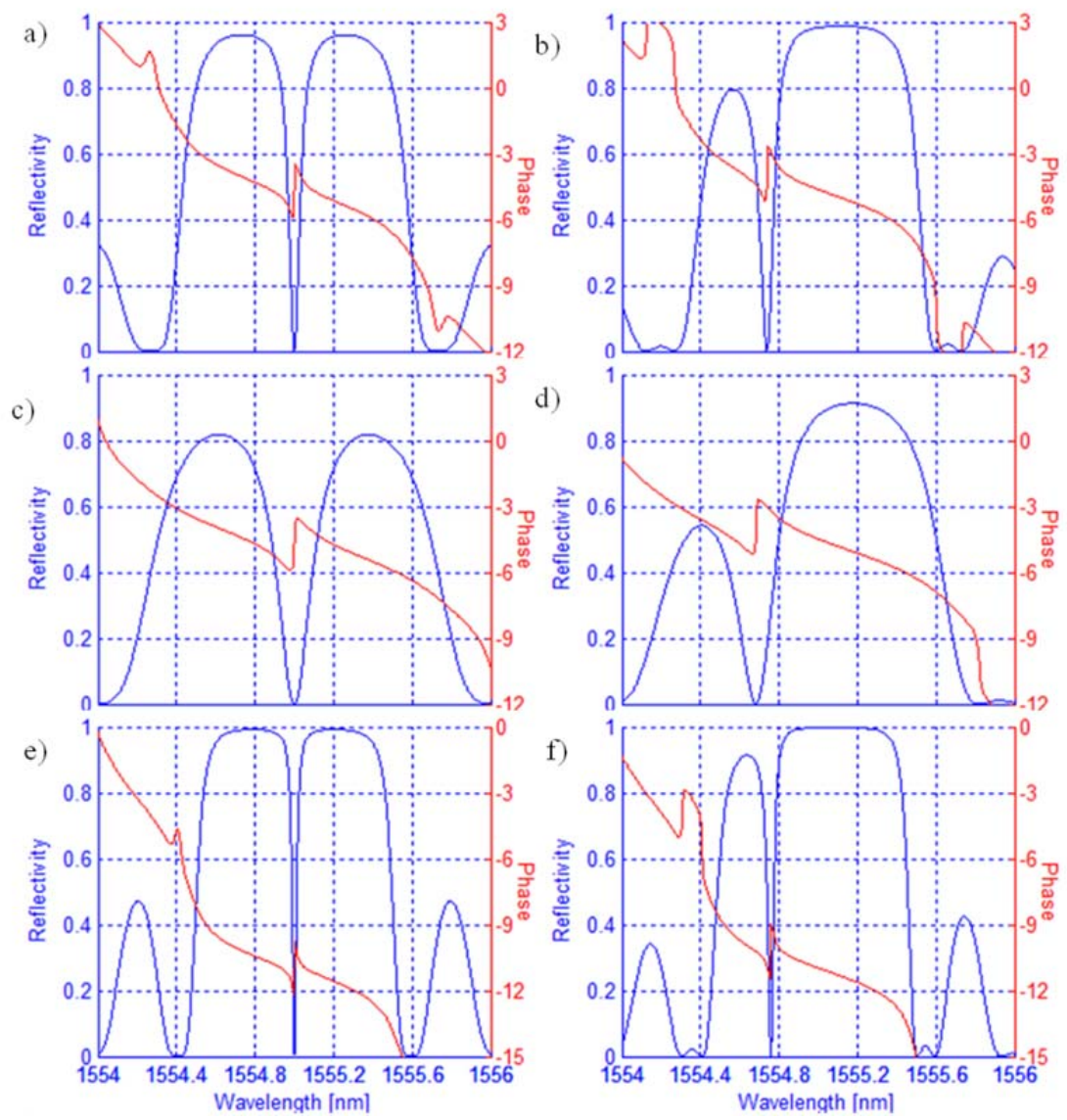

Figure 1. Reflectivity and phase response of the $\pi$ phase shifted FBG at different positions along the grating: (a) center, (c) $-\mathrm{L} / 6$, (e) $+\mathrm{L} / 6$. Reflectivity and phase response of the $\pi / 2$ phase shifted FBG in the center at different positions along the grating: (b) center, (d) $-\mathrm{L} / 6$, (f) $+\mathrm{L} / 6$.
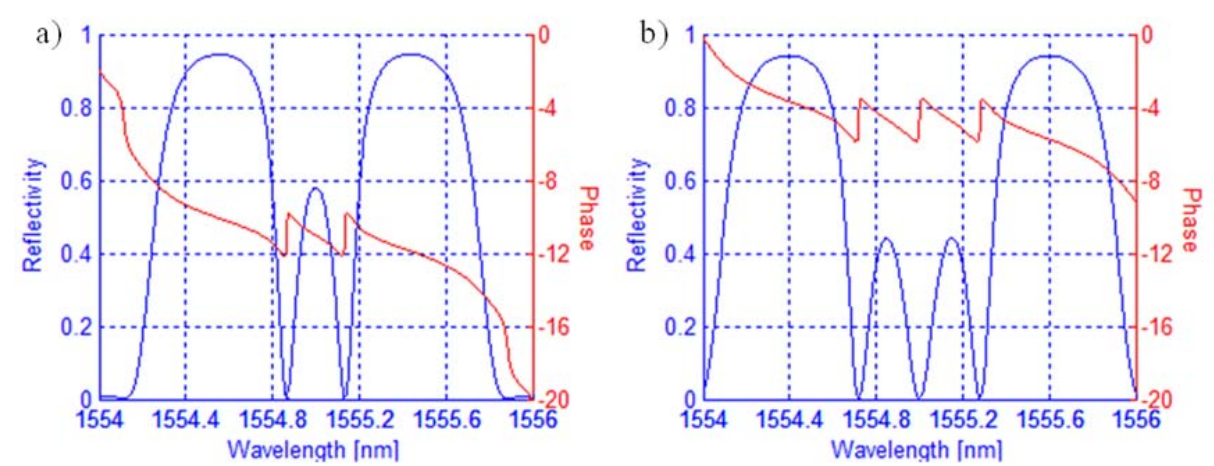

Figure 2. (a) Reflectivity and phase response of two $\pi$ phase shift FBG, (b) Reflectivity and phase response of the three $\pi$ phase shifts FBG. 


\subsection{Moiré grating}

The phase-shifted structure can also be reproduced as a Moiré grating where two gratings of equal amplitude but different periods, $\Lambda_{1}$ and $\Lambda_{1}$, are superimposed [34]. This structure has one rapidly, $\Lambda_{s}$, and one slowly, $\Lambda_{c}$, varying envelope, as shown in Figure 3. Thus, the index modulation change $\Delta \mathrm{n}(\mathrm{z})$ depends on the $\mathrm{z}$ coordinate along the axis of the fibre as follows [35]:

$$
\Delta n(z) \alpha\left(2+2 \cos \frac{2 \pi z}{\Lambda_{\mathrm{c}}} \cdot \cos \frac{2 \pi z}{\Lambda_{\mathrm{s}}}\right)
$$

Where both periods are obtained in terms of the original superimposed grating periods as given by the expressions:

$$
\begin{gathered}
\Lambda_{\mathrm{c}}=\frac{2 \Lambda_{1} \Lambda_{2}}{\Lambda_{1}-\Lambda_{2}} \\
\Lambda_{\mathrm{S}}=\frac{2 \Lambda_{1} \Lambda_{2}}{\Lambda_{1}+\Lambda_{2}}
\end{gathered}
$$

Provided the grating length equals $\Lambda_{\mathrm{C}}$, (see Figure 3), an intrinsic $\pi$ phase-shift is created in the central point of this structure. Other phase shift values different form $\pi$ can be achieved by superimposing gratings with different amplitudes and a large number of phase shifts are obtained in longer gratings, as will be demonstrated below in this paper.

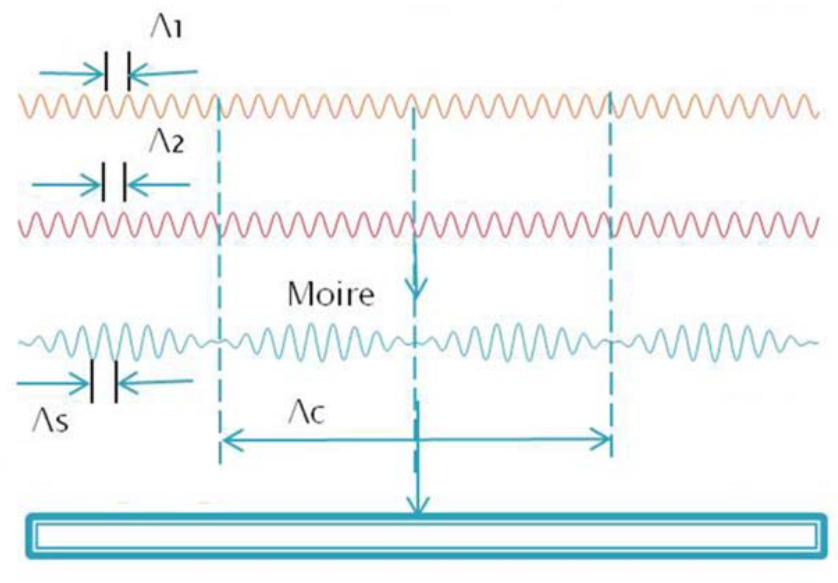

Figure 3. Moiré grating.

Figure 4 a) shows the reflectivity and phase response of a $2.5 \mathrm{~mm}$ Moiré long grating on polymer fiber (refractive index of 1.46) which length equals $\Lambda_{\mathrm{c}}$. The grating response shows the expected absolute equivalence of this structure and the one simulated in Figure 2a). Figure $4 b$ ) shows a Moiré grating $L=2 \Lambda_{c}$, similar to the response depicted in Figure 2b), which corresponds to three phase shifts inside the grating, as expected from Figure 3.
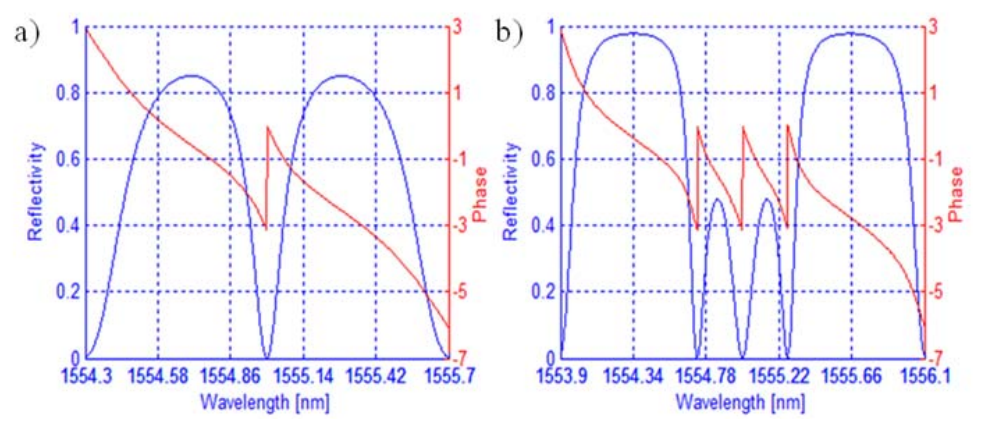

Figure 4 (a) Reflectivity and phase response of the Moiré grating when $L=\Lambda_{c}$, (b) Reflectivity and phase of the Moiré grating when $\mathrm{L}=2 \Lambda_{\mathrm{c}}$.

Figure 5 shows the response of the resulting structure from overlapping two gratings with different coupling coefficients, 
as happens during the second grating fabrication process and will be confirmed in the next section. For simulations, the refractive index change of the second grating has been considered as $1 / 4,1 / 2$ and $3 / 4$ ( $a, b$, and c, respectively) respect to the index change of the first grating. It is observed that the obtained phase shift varies for different simulations (i.e. wavelength shift towards lower values as long as the coupling coefficients of both gratings approach).
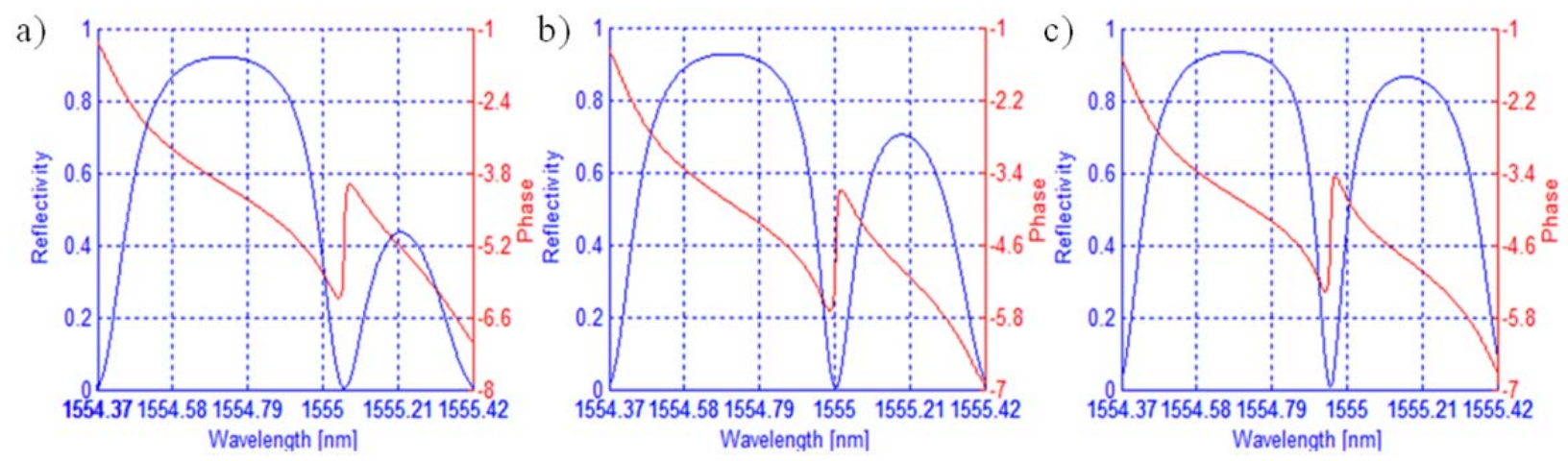

Figure 5. Reflectivity and phase response of the Moiré grating resulting by superimposing gratings with different relative index changes: (a) $1 / 4,(b) 1 / 2$, (c) $3 / 4$.

\section{PS-FBG FABRICATION}

In our setup, a $325 \mathrm{~nm}$ CW He-Cd laser (Kimmon IK3301R-G) emitting an output power of $32 \mathrm{~mW}$ was employed for grating inscription; the fiber was mounted horizontally between two v-grooves and the beam was focused onto the fiber using a horizontal cylindrical lens (see Figure 6a). A microstructured polymer fiber, mPOF, based on PMMA with a three ring hexagonal cladding structure with a relative hole diameter/pitch of 0.44 and NA=1.46 was employed (inset of Figure 6b) [29]. The UV light was passed through a $1066.2 \mathrm{~nm}$ pitch phase mask with a length of $2.5 \mathrm{~mm}$ onto the fiber. Initially, the fiber was subjected to a strain of $1 \%$, in order to increase the photosensitivity [29], and the first uniform grating was fabricated as a first step of the inscription process, then the fiber strain was changed to $1.0431 \%$ in order to slightly modify the grating pitch. Then, a second grating was written over the same length of the fiber in the second step of the fabrication process. Therefore, both gratings were spatially superimposed leading to a Moiré grating which exhibits a phase shift at the central point [34]. Other examples of dual FBG inscription can be found in the literature, such as spatially close [37] or spatially overlapped [38] polymer FBGs with different Bragg wavelengths.

a)

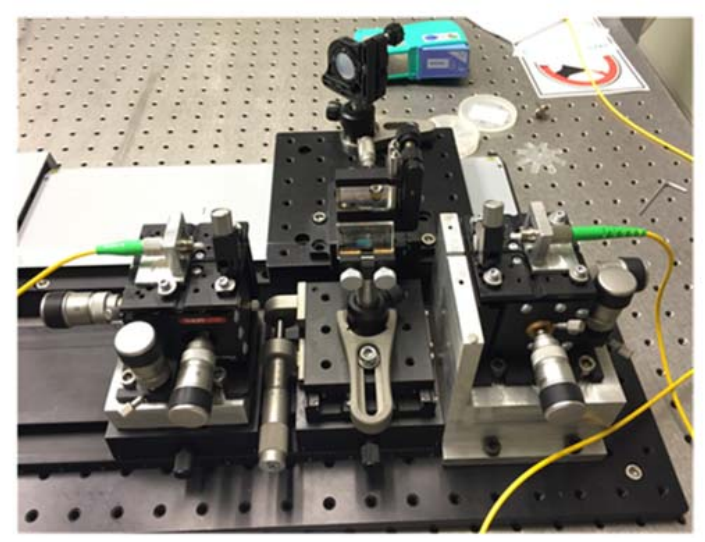

b)

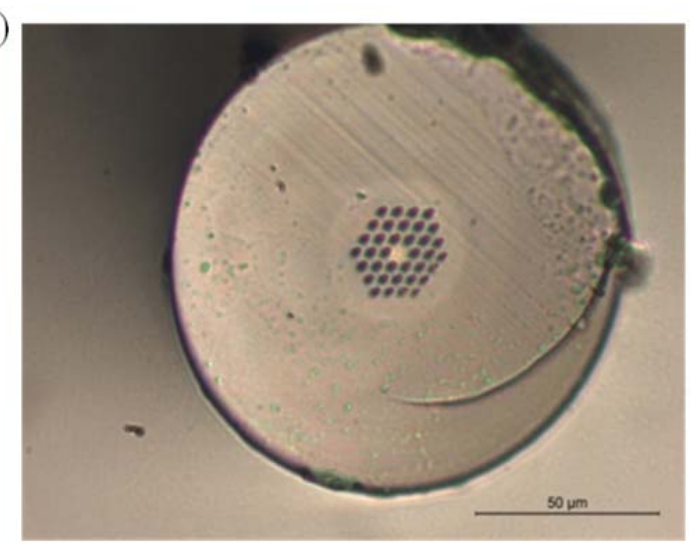

Figure 6 (a) Inscription setup and (b) Transversal section of the optical fiber.

Figure 7 shows the transmission response of three fabricated gratings (A, B and C) along the fabrication process. The uniform FBG grating fabricated in the first step of the inscription process is shown in Figure 7a) and after several 
minutes, the second superimposed grating started to be fabricated as detailed above. During the second step of the fabrication process, the transmission spectrum (Figure 7b) showed some phase shift, although not pi-phase shift was encountered (see Figure 5) due to the different index changes of both gratings. After stopping the fabrication process, the transmission spectrum showed a single transmission band corresponding to a uniform FBG (Figure 7c). However, when the laser was switched off, a clear pi-phase shift was found in the FBG transmission spectrum (Figure 7d) and repetitive experimental results confirm that PS-FBG were correctly being fabricated, although, longer exposure times lead to local heating which temporarily avoids to show the PS transmission characteristic. This is due to the high thermo-optic effect in polymers which is 10 times larger than in silica [20]. While the laser is on, the illuminated areas are being heated up and therefore, the refractive index modulation is higher and it results in a temporary strong second grating. When the laser is turned off, the temperature decreases and the grating becomes of a similar intensity to the first one, observing a $\pi$-phase shift.

Grating A
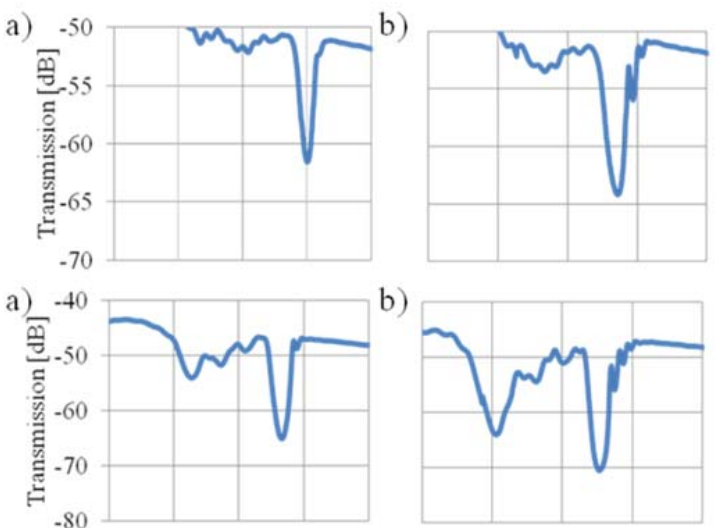

Grating B

$$
-80
$$

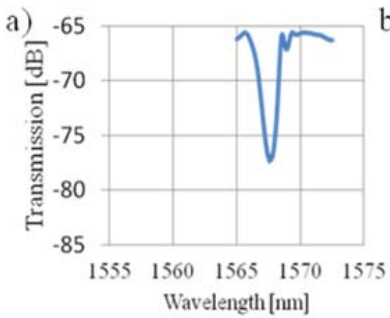

b)

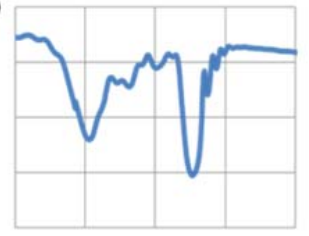

b)

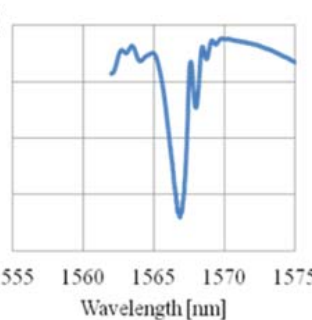

c)

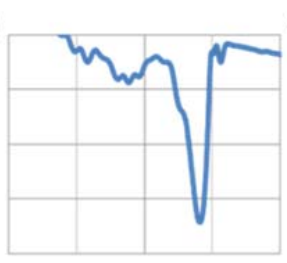

c)

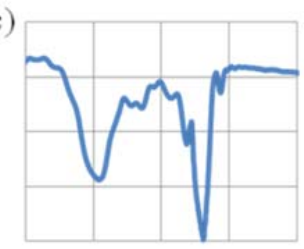

c)

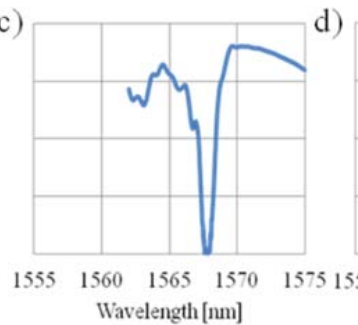

d)

d)

d)
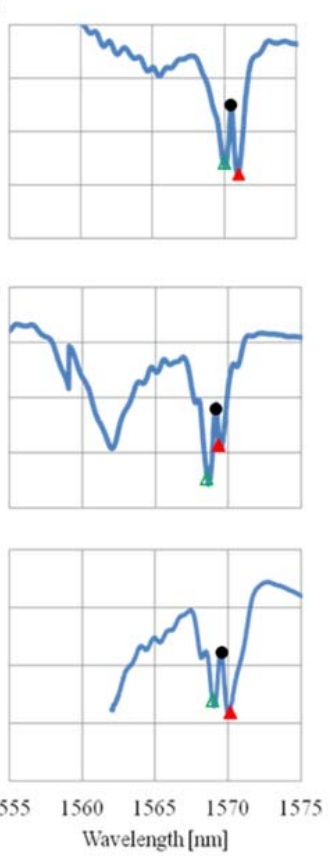

Figure 7. Transmission response of Fiber Bragg gratings fabricated along the process: a) Uniform FBG after first step fabrication, b) PS-FBG during the second step fabrication, c) PS-FBG after the inscription and d) FBG after fabrication when the laser is switched off.

Different results are due to the impact of the orientation in micro-structured fibers [39] which leads to strong variation on the intensity of the fabricated gratings. However, similar spectral characteristics are obtained in all cases.

Figure 8 allows to observe with more detail the evolution of the reflectivity and wavelength of both peaks and notch of the grating A response. The first step corresponds to the $2.5 \mathrm{~mm}$ long uniform grating fabrication, which corresponds to a maximum refractive index change of 0.0005 in 6 min inscription. Since that moment, the second overlapped grating is fabricated and different peaks can be distinguished. Reflectivity and wavelength of both peaks and notch are plotted at Figure 8 a) and b), respectively. At 8 min since the beginning of the fabrication process, a notch appears and two peaks are well defined which keep increasing until $14 \mathrm{~min}$. At $19 \mathrm{~min}$, no PS-FBG can be observed due to fiber heating but when the laser is switched off, both peaks can be distinguished, as described before.

Figure 9 depicts the experimental PS-FBG transmission spectrum obtained just after fabrication. A $11 \mathrm{~dB}$ stopband consisting of two bands is observed in the transmission spectrum with a total $3 \mathrm{~dB}$ bandwidth of $2.4 \mathrm{~nm}$ and a notch appears in the central wavelength with $5 \mathrm{~dB}$ insertion losses and a bandwidth around $0.2 \mathrm{~nm}$ at a $-3 \mathrm{~dB}$ level under its maximum transmission peak. Theoretical predictions calculated as detailed in section II are also plotted in the same graph and show a good fit to the obtained measurements. In this case, it is observed that a fiber with uniform refractive 
index along the z-axis leads to a narrower filter (solid line in Figure 9), whereas $0.135 \%$ difference in the refractive index along the grating, probably due to some imperfections in the fiber fabrication process (dotted line in Figure 9) leads to a good agreement between theoretical and experimental results.

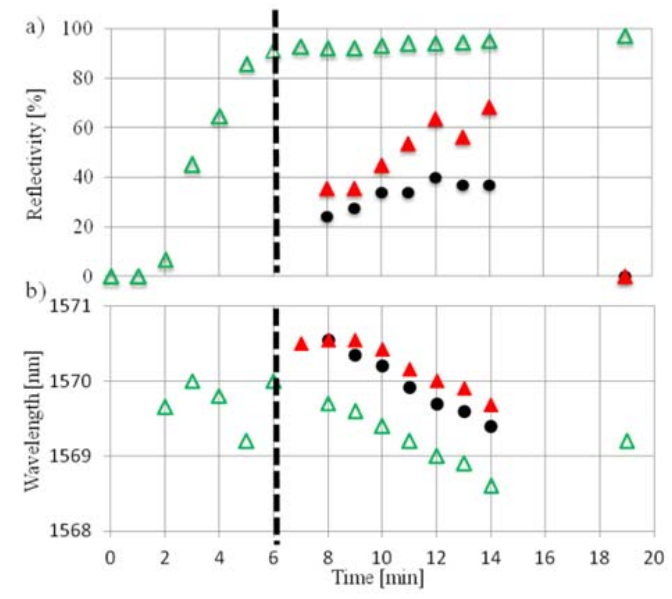

Figure 8. Bragg grating A characteristics during the fabrication process: a) FBG reflectivity at both peaks and notch and b) corresponding wavelengths ( $\triangle$ peak $1, \cdot$ notch and $\boldsymbol{\Delta}$ peak 2 ).

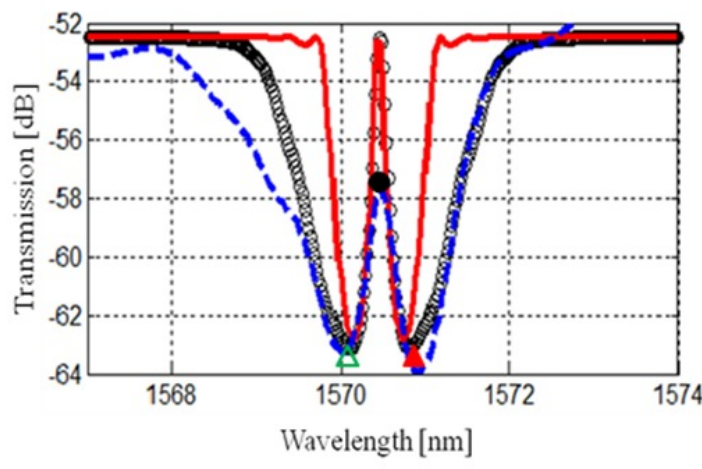

Figure 9. Transmission spectrum of the PS-FBG A: experimental (dashed line), simulations using non-uniform fiber (solid line) and using uniform fiber (dotted line).

Finally, as explained in section II, the Moiré approach for fabricating PS-FBG shows high degree of flexibility in terms of the grating structure to include a larger number of phase shifts. Figure 10 shows the experimental results of a $5 \mathrm{~mm}$ long grating with three phase shifts, as expected from theoretical predictions (see Figure 4b).

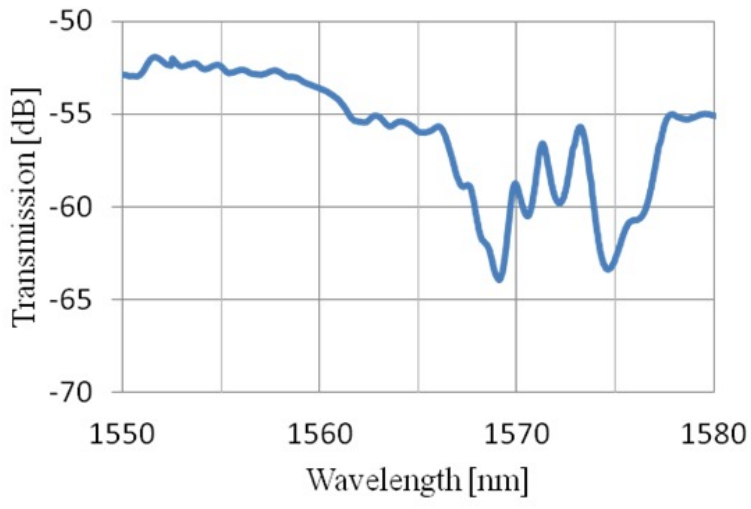

Figure 10. Transmission spectrum of the three PS-FBG.

\section{GRATING STABILITY}

Once the fabrication process is finished and grating B is released from the strain but suspended freely between two supports, the grating wavelength and reflectivity evolves with time according to the polymer material strain relaxation (see Figure 11) in the non-annealed employed fiber. After less than 4 hours, the phase shift cannot be observed any more. However, [40] shows that the relaxation time (i.e. stability) of FBG on polymers is affected by the viscoelastic behavior which depends on the time and the level of strain the fiber was stretched. Therefore, the experiment is completed by measuring the stability of the released grating response over time after a strain cycle (see section 5 of this paper) from 0 to $1.8 \%$ over 9 hours. In this case, as depicted in Figure 12, reflectivity and resonance wavelength are measured over 14 hours. We observe several fluctuations both in reflectivity and peak wavelengths over the first 5 hours but since then, wavelength and reflectivity stability are better than $0.25 \mathrm{~nm}$ and $3.25 \%$, respectively. A residual wavelength difference 
of $3.4 \mathrm{~nm}$ is observed with respect to the released grating just after fabrication (see Figure 7) due to polymer material hysteresis properties although this effect can be significantly reduced when the FBG is bonded directly to a substrate [41].
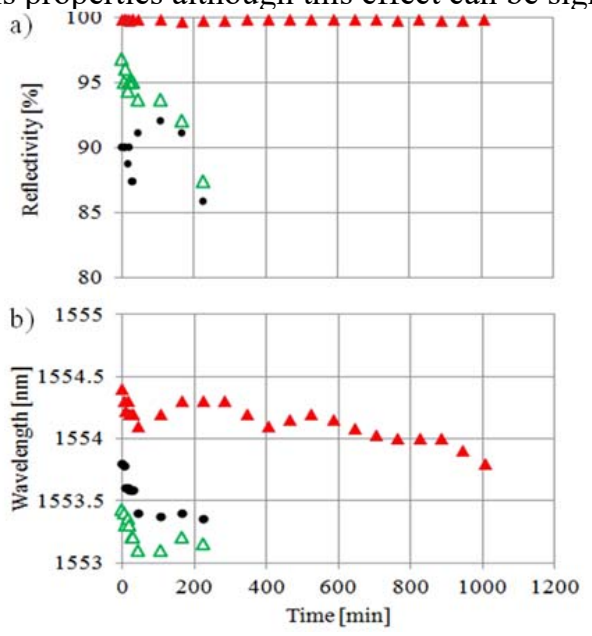

Figure 11. Grating B: Stability when released after fabrication $(\Delta$ peak $1, \cdot$ notch and $\Delta$ peak 2$)$ : a) reflectivity and b) wavelength.
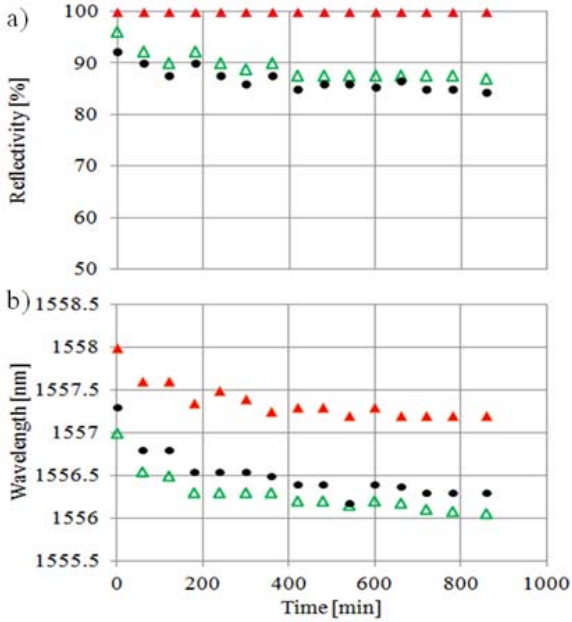

Figure 12. Grating B: Stability when released after strain experiment $(\Delta$ peak $1, \bullet$ notch and $\boldsymbol{\Delta}$ peak 2$)$ : a) reflectivity and $b$ ) wavelength.

\section{STRAIN AND TEMPERATURE CHARACTERIZATION}

The fabricated PS-FBG C has been tested under different strain and temperature conditions in order to characterize its stability in the short time regime.

\subsection{Strain}

The PS-FBG was subjected into an optical stage to strain the fiber from $0.2 \%$ up to $1.8 \%$, as a step function in time every 60 minutes at room temperature and we measured the wavelength and reflectivity for both peaks and notch of the PSFBG reflection spectrum, as plotted in Figure 13. It is important to notice that no PS can be observed with strains under $1 \%$ and some reflectivity variations are encountered for higher values of strain. Regarding wavelength, the measurements lead to a $1.58 \mathrm{pm} / \mathrm{m} \varepsilon$ sensitivity, which is somewhat higher to previous results in the literature [30], since fibers have not been annealed in this work.
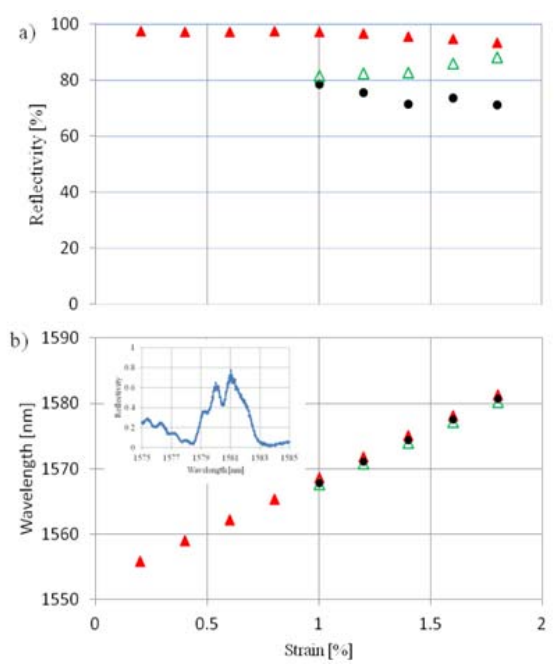

Figure 13. Grating C Sensitivity with strain ( $\triangle$ peak $1, \bullet$ notch and $\Delta$ peak 2): a) Reflectivity and b) Wavelength; inset: Reflectivity at $1.8 \%$ strain.
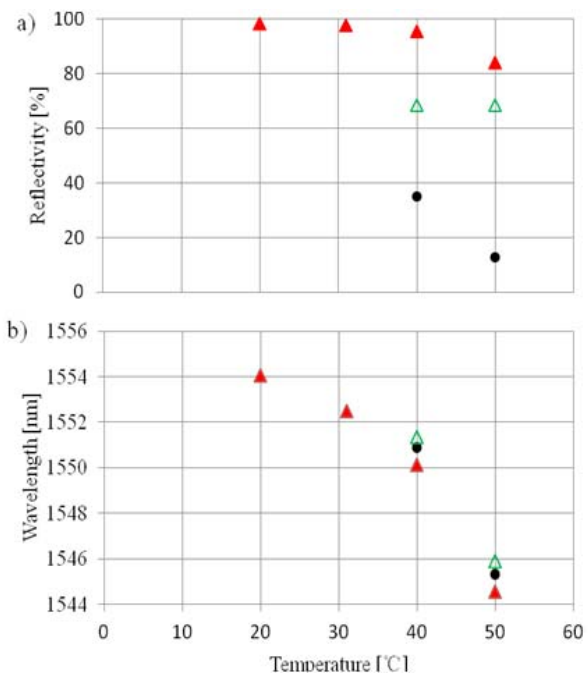

Figure 14. Grating C Sensitivity with temperature $(\Delta$ peak $1, \bullet$ notch and $\boldsymbol{\Delta}$ peak 2 ): a) Reflectivity and b) Wavelength. 


\subsection{Temperature}

Temperature was also varied as a step function in time from $20^{\circ} \mathrm{C}$ to $50^{\circ} \mathrm{C}$. As depicted in Figure 14 , in terms of wavelength, a total change of $30^{\circ}$ leads to $10 \mathrm{~nm}$ tuning but linear behaviour is only shown for temperatures up to $40^{\circ}$. The obtained wavelength shift is higher than expected from previous measurements [30] and measurements at $50{ }^{\circ} \mathrm{C}$ lead to strong variation in reflectivity and wavelength characteristics of the PS-FBG, which means some degradation of the phase shift is present and requires proper fiber annealing for further work.

\section{CONCLUSIONS}

In conclusion, for the first time to our knowledge, we demonstrate the fabrication of a PS-FBG in polymer optical fibers using the phase mask technique. We propose to obtain the phase shift by superimposing two quasi similar uniform fiber Bragg gratings to form a Moiré structure in a simple experimental setup. In this paper, theoretical and experimental demonstrations confirm the response of these structures which also allow the fabrication of broader transmission filters by employing multiple phase shifts structures. POF are very attractive for the deployment of current and future high bandwidth access networks and the phase shifted devices open new opportunities for telecommunication applications in DWDM networks and also for sensing and microwave photonic applications, where advantages of polymer fibers can be exploited in combination with high thermal and strain sensitivity characteristics. Experimental measurements of stability of the fabricated devices under different pre-strain conditions as well as their strain and temperature responses are also provided and lead to confirm that highly tunable polymer fiber devices have been developed although time stability of polymers needs to be correctly addressed prior to their practical usage.

\section{REFERENCES}

[1] N.F.Naim, M.S.Ab-Rahman, H.A. Bakarman, A. A. Bakar, "Real-time monitoring in passive optical networks using a superluminescent LED with uniform and phase-shifted fiber Bragg gratings", IEEE/OSA Journal of Optical Communications and Networking, vol. 5, 1425-1430 (2013).

[2] T.Y.Kim, M.Hanawa, S.J. Kim, S. Hann, Y.H. Kim, W.T. Han, C.S.Park, "Optical DPSK demodulator based on piphase-shifted fiber Bragg grating with an optically tunable phase shifter", IEEE Photonics Technology Letters, vol. $18,1834-1836(2006)$.

[3] Y. Lai, W. Zhang, L. Zhang, J. A. R. Williams, I. Bennion, “An optical pump controlled tunable phase-shifted fiber grating transmission filter", Optical Fiber Comunication Conference, OFC Proceedings, 35-37 (2003).

[4] A.Melloni, M.Chinello, M.Martinelli, "All-optical switching in phase-shifted fiber Bragg grating", IEEE Photonics Technology Letters, vol. 12, $42-44$ (2000).

[5] J. Guo, C. Yang. "Highly stabilized phase-shifted fiber Bragg grating sensing system for ultrasonic detection." IEEE Photonics Technology Letters, vol. 27, 848-851(2015).

[6] A. Rosenthal, D. Razansky, V. Ntziachristos. "High-sensitivity compact ultrasonic detector based on a pi-phaseshifted fiber Bragg grating", Optics letters vol. 36, 1833-1835 (2011).

[7] Q. Zhang, N. Liu, T. Fink, H. Li, W. Peng, M. Han, "Fiber-optic pressure sensor based on-phase-shifted fiber Bragg grating on side-hole fiber" IEEE Photonics Technology Letters, vol. 24, 1519-1522 (2012).

[8] X.Han, J.Yao, "Bandstop-to-bandpass microwave photonic filter using a phase-shifted fiber Bragg grating", Journal of Lightwave Technology, vol. 33, 5135-5139 (2015).

[9] O.Xu, Jiejun Zhang, H.Deng, J.Yao, "Dual-frequency optoelectronic oscillator for thermal-insensitive interrogation of a FBG strain sensor", IEEE Photonics Technology Letters, vol. 29, 357-360 (2017).

[10] G.P. Agrawal, S.Radjic, "Phase-shifted fiber Bragg gratings and their application for wavelength demultiplexing", IEEE Photonics Technology Letters, vol. 6, 995-997 (1994).

[11] C. Marra, A. Nirmalathas, D. Novak, C. Lim, L. Reekie, J. A. Besley, C. Weeks, N. Baker, "Wavelength-Interleaved OADMs Incorporating Optimized Multiple Phase-Shifted FBGsfor Fiber-Radio Systems", Journal of Lightwave Technology, vol. 21, 32-39 (2003).

[12] Li, Ming, Takeo Fujii, and Hongpu Li. "Multiplication of a multichannel notch filter based on a phase-shifted phaseonly sampled fiber Bragg grating." IEEE Photonics Technology Letters, vol. 21(13), 926-928 (2009).

[13] M.Joncic, R.Kruglov, M.Haupt, R.Caspary, J.Vinogradov, U.H.P. Fischer, "Four-channel WDM transmission over $50 \mathrm{~m}$ SI-POF at $14.77 \mathrm{~Gb} / \mathrm{s}$ using DMT modulation", IEEE Photonics Technology Letters, vol.26,1328-1331 (2014).

[14] G. Rajan, B. Liu,Y. Luo, E. Ambikairajah, G.D. Peng, "High Sensitivity force and pressure measurements using etched singlemode polymer fiber bragg grating" IEEE Sensors Journal 13, 1794-1800 (2013). 
[15]C. Zhang, W. Zhang, D.J. Webb, G.D. Peng, "Optical fibre temperature and humidity sensor", Electronics Letters, vol. 46, 643-644 (2010).

[16] G. Emiliyanov, P.E. Hoiby, L.H. Pedersen, O. Bang, "Selective serial multi-antibody biosensing with TOPAS microstructured polymer optical fibers", Sensors, vol. 13, 3242-3251 (2013)

[17] O. Ziemann and H. Poisel, "Short distance optical connections for home networks, sensing and mobile systems" OFC (2007).

[18] Y.Koike, T.lshigure, "Progress of low-loss GI polymer optical fiber from visible to 1.5-um wavelength", European Conference on Optical Communications ECOC 97 (1997).

[19]R.Kruglov, J.Vinogradov, S.Loquai, O.Ziemann, C.A.Bunge, T.Hager, U.Strauss, "21.4 Gb/s discrete multitone transmission over 50 m SI-POF employing 6-channel WDM", Optical Fiber Communications Conference, paper Th2A.2 (2014).

[20]P.J.Pinzon, I.Perez-Garcilopez, C.Vazquez, "Efficient multiplexer/demultiplexer for visible WDM transmission over SI-POF Technology", IEEE Journal of Lightwave Technology, vol. 33, 3711-3718 (2015).

[21]R.Caspary, M.Joncic, J.Haupt, U.Fischer, R.Fruglov, J.Vinogradov, H.H. Johannes, W. Kowalsky, "High speed WDM transmission on standard polymer optical fibers", ICTON, paper Tu.B1.2 (2015).

[22]Z.Xiong, G.D.Peng, B.Wu and P.L.Chu, "Highly tunable Bragg gratings in single-mode polymer optical fibers", IEEE Photonics Technology Letters, vol. 11, 352-354 (1999).

[23] K.Bhowmik, G.D.Peng, Y.Luo, E.Ambikairajah, V.Lovric, W.R.Walsh, G.Rajan, "High intrinsic sensitivity etched polymer fiber Bragg grating pair for simultaneous strain and temperature measurements", IEEE Sensors Journal, vol. $16,2453-2459$ (2016).

[24] G. Woyessa, A. Fasano, A. Stefani, C. Markos, K. Nielsen, H.K. Rasmussen, O. Bang, "Single mode step-index polymer optical fiber for humidity insensitive high temperature fiber Bragg grating sensors", Optics Express, vol. 24, 1253-1260 (2016).

[25] J-M.Yu, X-M.Tao, H-Y.Tam, “Trans-4-stilbenemethanol- doped photosensitive polymer fibers and gratings“, Optics Letters, vol. 29, 156-158 (2004).

[26] C.Markos, A.Stefani, K.Nielsen, H.Rasmussen, W.Yuan, O. Bang, "High-Tg TOPAS microstructured polymer optical fiber for fiber Bragg grating strain sensing at 110 degrees", Optics Express, vol. 21, 4758-4765 (2013).

[27] Y.Luo, Q.Zhang, H.Liu, G.D.Peng, "Gratings fabrication in benzildimethylketal doped photosensitive polymer optical fibers using $355 \mathrm{~nm}$ nanosecond pulsed laser", Optics Letters, vol. 35,751-753 (2010).

[28] D.Sáez-Rodríguez, K.Nielsen, H.Rasmussen, O.Bang, D.J.Webb, "Highly photosensitive polymethyl methacrylate microstructured polymer optical fiber with doped core", Optics Letters, vol. 38, 3769-3772 (2013).

[29] D.Sáez-Rodríguez, K.Nielsen, O.Bang, D.J.Webb, "Photosensitivity mechanism of undoped poly(methyl methacrylate) under UV radiation at $325 \mathrm{~nm}$ and its spatial resolution limit", Optics Letters, vol. 39, 3421-3424 (2014).

[30] W. Yuan, A. Stefani, M. Bache, T. Jacobsen, B. Rose, N. Herholdt-Rasmussen, F.K. Nielsen, S. Andresen, O.B. Sørensen, K.S. Hansen, O. Bang, "Improved thermal and strain performance of annealed polymer optical fiber Bragg gratings", Optics Communications, vol. 284(1), 176-182 (2011).

[31] X. Hu, D. Kinet, P. Megret, C. Caucheteur, "Control over photo-inscription and thermal annealing to obtain highquality Bragg gratings in doped PMMA optical fibers", Optics Letters, vol. 41 2930-2933(2016)

[32] C.A.F.Marques, P.Antunes, P.Mergo, D.J.Webb, P.Amdre, "Chirped Bragg gratings in PMMA step-index polymer optical fiber", IEEE Photonics Technology Letters, vol. 29, 500-503 (2017).

[33] S.F.Zhou, L.Reekie, Y.T.Chow, H.P.Chan, K.M.Luk, "Phase-shifted fiber Bragg gratings for Terahertz range", IEEE Photonics Technology Letters, vol. 24, 1875-1877 (2012).

[34]D.C.J.Reid, C.M.Ragdale, I.Bennion, D.J.Robbins, J.Buus, W.J.Stewart, "Phase shifted Moiré grating fibre resonators", Electronics Letters, vol. 26, 10-12 (1990).

[35] S.Legoubin, E.Fertein, M.Douay, P.Bernange, P.Niay, "Formation of Moire grating in core of germanosilicatefibre by transverse holographic double exposure method", Electronics Letters, vol. 27,1945-1947 (1991).

[36] T. Erdogan. "Fiber gratings spectra”, Journal of Lightwave Technology, vol. 15, 1277-1294 (1997).

[37] W. Yuan, A. Stefani, O. Bang, "Tunable polymer Fiber Bragg Grating (FBG) inscription: Fabrication of dual-FBG temperature compensated polymer optical fiber strain sensors," IEEE Photonics Technology Letters, vol. 24, 401403 (2012).

[38] D. Sáez-Rodríguez, K. Nielsen, O. Bang, B. Ortega, "Compact multichannel demultiplexer for WDM-POF networks based on spatially overlapped FBGs", Electronics Letters, vol. 52, 635-637 (2016) 
[39] I.L. Bundalo, K. Nielsen, O. Bang, "Angle dependent fiber bragg grating inscription in microstructured polymer optical fibers”, Optics Express, vol. 23, 3699-3707 (2015).

[40] D.Sáez-Rodríguez, K.Nielsen, O.Bang, D.J.Webb, "Time-dependent variation of Fibre Bragg Grating Reflectivity in PMMA based polymer optical fibres", Optics Letters, vol. 40, 1476-1479 (2015).

[41] A.Abang, D.J.Webb, "Influence of mounting on the hysteresis of polymer fiber Bragg grating strain sensors", Optics Letters, vol. 38, 1376-1378 (2013). 